\title{
A NOTE ON THE HEAT KERNEL METHOD APPLIED TO FERMIONS
}

\author{
G. DE BERREDO-PEIXOTO円 \\ Department of Physics, University of Alberta \\ 412 Avadh Bhatia Physics Laboratory, Edmonton, AB, T6G 2J1 Canada. \\ Instituto de Física, Universidade de Brasília \\ Brasília, DF, 70910-900 Brazil;
}

\begin{abstract}
The spectrum of the fermionic operators depending on external fields is an important object in quantum field theory. In this paper we prove, using transition to the alternative basis for the $\gamma$-matrices, that this spectrum does not depend on the sign of the fermion mass, up to a constant factor. This assumption has been extensively used, but usually without proof. As an illustration, we calculated the coincidence limit of the coefficient $a_{2}\left(x, x^{\prime}\right)$ on the general background of metric, vector and axial vector fields.
\end{abstract}

\section{Introduction}

In four space-time dimensions, a relevant part of the spectrum of a differential operator is the coincidence limit of the coefficient ${ }^{2} a_{2}\left(x, x^{\prime}\right)$ in the Schwinger-DeWitt expansion [1]. This coefficient defines the divergent part of the effective action and is closely related to the anomalies. The calculation of the differential operator spectrum is a mathematical problem with important applications in theoretical physics; it defines the relation between matter fields and quantum corrections to the geometry (for general introduction, see [2, 30). Among various papers that investigate these physical applications, we can cite [4, 5, 6, 7, 8, 9, 10, 11, 12]. One can mention that the papers [4, 5, 6, 6, 8, 9, 10] consider torsion, which gives the general coupling between geometry and fermions (see also [3] and [13]). The calculation of the determinant of a differential operator corresponding to some particular matter field is a necessary tool to investigate the effective action at the 1-loop level. In many cases, the 1-loop contribution contains the most relevant information of the quantum effects in the low energy regime. The 1-loop part of the effective action can be written as (see [3] )

$$
\Gamma^{(1)}[\phi]=\epsilon \frac{i}{2} \ln \operatorname{det} S_{2}[\phi]=\epsilon \frac{i}{2} \operatorname{Tr} \ln S_{2}[\phi]
$$

where $\phi$ is an arbitrary set of background fields (mean fields), $\hat{H}=S_{2}[\phi]$ is the bilinear functional operator $\delta^{2} S[\phi] / \delta \phi(x) \delta \phi(y)$ and $\epsilon=+1$ for bosonic fields and $\epsilon=-1$ for fermionic fields. One can use the Schwinger-DeWitt method of proper time expansion to calculate the determinant (11). The heat kernel approach has been widely developed for many applications in Physics, as well as a tool of mathematical nature (see, for example, [14] and references therein). For instance, Goldthorpe [4] considered a minimal second order operator in a Riemann-Cartan background, and calculated the asymptotic expansion coefficient, $a_{2}$, considering contributions from the skew-symmetric torsion. Nieh and Yan [7] used also the proper-time expansion technique to compute the coefficient, but they considered in addition all the invariants from the generic torsion as well as the conformal and axial anomalies. Obukhov [6] has generalized the Goldthorpe's result, by the use of the Seeley's method [15], finding the corresponding anomalies.

The first-order Dirac operator includes negative eigenvalues in its spectrum (see [12, 16]), so that one has to use a supposition about the evenness in the mass structure of the spectrum and to introduce the conjugated operator in order to make the application of the Schwinger-DeWitt

\footnotetext{
${ }^{1}$ Electronic address: gpeixoto@phys.ualberta.ca

${ }^{2}$ The coefficients $a_{i}$ are also called the Hadamard-Minackshisundaram-Seeley-DeWitt coefficients
} 
method possible (see [17, 12, 11]). The main purpose of the present paper is to prove the required assumption, which is universaly accepted by the authors who calculate the spectrum of the Dirac operator. The 1-loop effective action (11) corresponding to the Dirac action,

$$
S_{1 / 2}=i \int d^{4} x \sqrt{-g} \bar{\psi}\left[\gamma^{\mu} D_{\mu}-i m\right] \psi
$$

is

$$
\Gamma^{(1)}[\phi]=-i \operatorname{Tr} \ln \{\hat{H}[\phi]\}
$$

where

$$
\hat{H}=i \gamma^{\mu} D_{\mu}+m,
$$

and $g$ is the metric determinant. Here $D_{\mu}$ is the covariant derivative, which includes usual couplings:

$$
D_{\mu}=\nabla_{\mu}-i e A_{\mu}+i \eta \gamma^{5} S_{\mu},
$$

where $e$ and $\eta$ are dimensionless coupling constants and $\nabla_{\mu}$ is the covariant derivative in the curved background without torsion, as defined in [13].

This paper is organized as follows. In section 2, we review the basic concepts and calculations widely assumed in the spectral theory applied to the Dirac operator. As an illustration, we present the general result for the 1-loop divergence calculation in the Dirac action in the background Abelian vector, torsion and metric fields, including the superficial terms which are useful for the axial and trace anomaly calculation. In section 3 , we demonstrate that a special assumption pointed out in section 2 is true.

\section{Fermionic Operator coupled to External Fields}

In order to use the Schwinger-DeWitt proper-time technique, the following trick is assumed and applied to the calculation of the 1-loop divergences in Dirac theories on curved backgrounds (see [11, 12, 16]):

$$
-i \operatorname{Tr} \ln \left\{i \gamma^{\mu} D_{\mu}+m\right\}=-\frac{i}{2} \operatorname{Tr} \ln \left\{\gamma^{\mu} D_{\mu} \gamma^{\nu} D_{\nu}+m^{2}\right\}+C ;
$$

where $C$, which is formally divergent, does not depend on the background quantities, so it is not relevant for physical considerations ${ }^{a}$. Basically, it arises here from the contribution of the term $C_{0}$ in the following relation (which is required to prove (6) $)^{\text {f }}$ :

$$
-i \operatorname{Tr} \ln \left(i \gamma^{\mu} D_{\mu}+m\right)=-i \operatorname{Tr} \ln \left(i \gamma^{\mu} D_{\mu}-m\right)+C_{0} .
$$

Here $C_{0}$ is divergent and constant (see (12) and (16) below). By straightforward calculation, one can write the second order operator in the right hand side of (6) in the useful form

$$
\square+R_{\mu} \nabla^{\mu}+\Pi
$$

where

$$
\begin{aligned}
R_{\mu} & =-2 i e A_{\mu}+2 \eta \Sigma_{\mu \nu} \gamma^{5} S^{\nu} \\
\Pi & =-i e \nabla^{\mu} A_{\mu}+i \eta \gamma^{5} \nabla^{\mu} S_{\mu}-e^{2} A^{\mu} A_{\mu}+\eta^{2} S^{\mu} S_{\mu}-\frac{i}{2} e \gamma^{\mu} \gamma^{\nu} F_{\mu \nu}+ \\
& +\frac{i}{2} \eta \gamma^{\mu} \gamma^{\nu} \gamma^{5} S_{\mu \nu}-2 i e \eta \Sigma^{\mu \nu} \gamma^{5} A_{\mu} S_{\nu}-\frac{1}{4} R+m^{2}
\end{aligned}
$$

\footnotetext{
${ }^{3} A_{\mu}$ is the Abelian vector field, and $S_{\mu}$ is an axial vector field, which can be related to torsion in the RiemannCartan framework. Here $\gamma^{5}=i \gamma^{0} \gamma^{1} \gamma^{2} \gamma^{3}$

${ }^{a}$ The dimensional regularization scheme is adopted to deal with the important divergent quantities.

${ }^{b}$ Additionaly, a reparametrization of the (independent) fields, $(\psi, \bar{\psi}) \rightarrow(i \psi, i \bar{\psi})$ is done in order to arrive at ( 6 ) from (7), and, as the Jacobian is constant, this procedure produces a constant quantity, divergent, which is absorbed by $C$.
} 
with

$$
\begin{gathered}
\frac{1}{2} \gamma^{\mu} \gamma^{\nu}\left[\nabla_{\mu}, \nabla_{\nu}\right]=\frac{1}{8} \gamma^{\mu} \gamma^{\nu} \gamma^{\rho} \gamma^{\lambda} R_{\mu \nu \rho \lambda}=-\frac{1}{4} R \\
F_{\mu \nu}=\nabla_{\mu} A_{\nu}-\nabla_{\nu} A_{\mu}, \quad S_{\mu \nu}=\nabla_{\mu} S_{\nu}-\nabla_{\nu} S_{\mu} \text { and } \Sigma_{\mu \nu}=i / 2\left[\gamma_{\mu}, \gamma_{\nu}\right] .
\end{gathered}
$$

The object $R_{\mu \nu \alpha \beta}$ is the curvature tensor, $R_{\beta \mu \nu}^{\alpha}=\partial_{\mu} \Gamma_{\beta \nu}^{\alpha}+\Gamma_{\beta \nu}^{\lambda} \Gamma_{\lambda \mu}^{\alpha}-(\mu \leftrightarrow \nu)$ and $R=$ $g^{\mu \nu} R_{\mu \alpha \nu}^{\alpha}$. One can find the resulting expression for the corresponding 1-loop divergences, by direct use of theSchwinger-DeWitt algorithm:

$$
\begin{aligned}
\Gamma_{\mathrm{div}}^{(1)} & =\frac{\mu^{(n-4)}}{\varepsilon} \int d^{n} x \sqrt{-g}\left\{\frac{2}{3} e^{2} F_{\mu \nu}^{2}+\frac{2}{3} \eta^{2} S_{\mu \nu}^{2}-8 m^{2} \eta^{2} S_{\mu} S^{\mu}-\frac{1}{3} m^{2} R+2 m^{4}+\right. \\
& +\frac{1}{72} R^{2}-\frac{1}{45} R_{\mu \nu}^{2}-\frac{7}{360} R_{\mu \nu \rho \lambda}^{2}-\frac{4}{3} \eta^{2} \square\left(S^{\mu} S_{\mu}\right)+ \\
& \left.+\frac{4}{3} \nabla_{\mu}\left(S^{\nu} \nabla_{\nu} S^{\mu}-S^{\mu} \nabla_{\nu} S^{\nu}\right)-\frac{1}{30} \square R\right\} .
\end{aligned}
$$

\section{The Evenness in the Mass Structure of the Operator $i \gamma^{\mu} D_{\mu}+m$}

We are going to prove the relation (7), and as a byproduct, the validity of eq. (6). For this purpose we shall find a basis of the $\gamma$-matrices, in which the correctness of (6) becomes obvious. Following [18], let us consider the Dirac matrices basis, $\left(\Gamma^{\mu}, \Gamma^{4}\right)$, defined by:

$$
\Gamma^{\mu}=i \gamma^{5} \gamma^{\mu} ; \quad \Gamma^{4}=i \gamma^{5} .
$$

Notice that these matrices satisfy the Clifford algebra:

$$
\left\{\Gamma^{\mu}, \Gamma^{\nu}\right\}=2 g^{\mu \nu} \quad \text { and } \quad \Gamma^{4} \Gamma^{4}=-1 .
$$

Consider the operator $\hat{F}=\Gamma^{4} \hat{D}=\Gamma^{4}\left(i \Gamma^{\mu} D_{\mu}+m\right)$. The expression $-i \operatorname{Tr} \ln (\hat{F})$ is physically equivalent to $-i \operatorname{Tr} \ln \left(i \gamma^{\mu} D_{\mu}+m\right)$, because this quantity must not depend on which basis we choose, and the difference is a constant factor which is not relevant. One can write then

$$
-\frac{i}{2} \operatorname{Tr} \ln \left(\hat{F}^{2}\right)=-i \operatorname{Tr} \ln (\hat{F})=-i \operatorname{Tr} \ln \left(i \Gamma^{\mu} D_{\mu}+m\right)-i \operatorname{Tr} \ln \Gamma^{4} .
$$

One can find, by direct computation,

$$
\begin{aligned}
\Gamma^{\mu} D_{\mu} \Gamma^{\nu} D_{\nu} & =\Gamma^{\mu} \Gamma^{\nu}\left(\nabla_{\mu} \nabla_{\nu}-i e \nabla_{\mu} A_{\nu}-i e A_{\nu} \nabla_{\mu}-i e A_{\mu} \nabla_{\nu}+\right. \\
& +\eta \Gamma^{4} \nabla_{\mu} S_{\nu}+\eta \Gamma^{4} S_{\nu} \nabla_{\mu}-e^{2} A_{\mu} A_{\nu}-i e \eta \Gamma^{4} A_{\mu} S_{\nu}+ \\
& \left.+i e \eta \Gamma^{4} A_{\nu} S_{\mu}-\eta \Gamma^{4} S_{\mu} \nabla_{\nu}+\eta^{2} S_{\mu} S_{\nu}\right) .
\end{aligned}
$$

Using this expression to write down $\hat{F}^{2}$, and turning back to the usual basis, we arrive at the surprising result:

$$
\hat{F}^{2}=\square+R_{\mu} \nabla^{\mu}+\Pi,
$$

with $R_{\mu}$ and $\Pi$ exactly the same defined by (9). The conclusion is that

$$
\hat{F}^{2}=\hat{H}=\left(i \gamma^{\mu} D_{\mu}+m\right) \cdot\left(i \gamma^{\mu} D_{\mu}-m\right)
$$

Thus, we achieved that

$$
\begin{aligned}
-i \operatorname{Tr} \ln \hat{F} & =-i \operatorname{Tr} \ln \left(i \gamma^{\mu} D_{\mu}+m\right)+C_{0}=-\frac{i}{2} \operatorname{Tr} \ln \hat{F}^{2}= \\
& =-\frac{i}{2} \operatorname{Tr} \ln \left\{\left(i \gamma^{\mu} D_{\mu}+m\right) \cdot\left(i \gamma^{\mu} D_{\mu}-m\right)\right\} .
\end{aligned}
$$

which proves the desired result, eq. (16). By the virtue of the result (14) it is clear that one does not need to repeat the whole calculation done in the previous basis in section 2 and led to (10). Indeed, all the algebra will be the same and the new basis is as good as the original one, for both satisfy the same Clifford algebra. 


\section{Conclusion}

We have proved the assumption concerning the parity in the mass term, for the case of the most general Dirac operator in external fields. As an application, the divergent part of the fermion contribution to the vacuum effective action has been derived.

\section{Acknowledgments.}

I would like to thank Prof. Ilya L. Shapiro for suggesting me to study the alternative basis for fermions and for the fruitful discussions of the result. I am also grateful to Centro Brasileiro de Pesquisas Físicas. This work was done with support from CNPq, a Brazilian Government institution that provides development in science and technology.

\section{References}

[1] B.S. DeWitt, Dynamical theory of groups and fields (Gordon and Breach, New York, 1965).

[2] N.D. Birrell and P.C.W. Davies, Quantum Fields in Curved Space, Cambridge Univ. Press, Cambridge (1982).

[3] I.L. Buchbinder, S.D. Odintsov and I.L. Shapiro, Effective Action in Quantum Gravity, IOP Publishing-Bristol (1992).

[4] W.H. Goldthorpe, Spectral geometry and SO(4) gravity in a Riemann-Cartan spacetime, Nucl. Phys. B 170 (1980) 307-328.

[5] T. Kimura, Expansion coefficient of heat kernel of Laplacian operator in Riemann-Cartan space, J. Phys. A: Math. Gen. 14 (1981) 329.

T. Kimura, Conformal and axial anomalies in Riemann-Cartan space, Prog. Theor. Phys. 66 (1981) 2011.

[6] Yu.N. Obukhov, Spectral geometry of the Riemann-Cartan spacetime, Nucl. Phys. B 212 (1983) 237-254;

Yu.N. Obukhov, Spectral geometry of the Riemann-Cartan spacetime and the axial anomaly, Phys. Lett. B 108 (1982) 308-310;

[7] H.T. Nieh and M.L. Yan, Quantized Dirac field in curved Riemann-Cartan background. I. Symmetry properties, Green's function, Ann. of Phys. 138 (1982) 237-259.

[8] I.L. Buchbinder, S.D. Odintsov and I.L. Shapiro, Nonsingular cosmological model with torsion induced by vacuum quantum effects, Phys. Lett. B 162 (1985) 92.

[9] G. Cognola and S. Zerbini, Seeley-DeWitt coefficients in a Riemann-Cartan space-time, Phys. Lett. B 214 (1988) 70;

Heat kernel expansion in geometric fields, Phys. Lett. B 195 (1889) 435.

[10] S. Yajima, Evaluation of the heat kernel in Riemann-Cartan space, Class. Quant. Grav. 13 (1996) 2423-2435.

[11] D.G.C. McKeon and C. Schubert, Phys. Lett. B 440 (1998) 101.

[12] E. Elizalde, On the concept of determinant for the differential operators of Quantum Physics, JHEP 07: 015 (1999).

[13] I.L. Shapiro, Physical aspects of the space-time torsion. (to appear in Phys. Rep.) hepth/1013093.

[14] I.G. Avramidi, Covariant techniques for computation of the heat kernel, Rev. Math. Phys. 11 (1999) 947-980.

[15] R.T. Seeley, Proc. Symp. Pure Math., Amer. Math. Soc. 10 (1967) 288. 
[16] G. Cognola, E. Elizalde and S. Zerbini, Dirac Functional Determinants in Terms of the Eta Invariant and the Noncommutative Residue, hep-th/9910038.

[17] E. Abdalla, M.C.B. Abdalla and K.D. Rothe, 2-Dimensional Quantum Field Theory, World Scientific, Singapore (1991).

[18] B. Geyer, D. Gitman and I.L. Shapiro, Path integral and pseudoclassical action for spinning particle in external electromagnetic and torsion fields, Int. J. Mod. Phys. A 15 (2000) 38613876 . 